\title{
A SIMPLE PATH TRACKING CONTROLLER FOR CAR-LIKE MOBILE ROBOTS
}

\author{
André KAMGA and Ahmed RACHID \\ Laboratoire des Systèmes Automatiques \\ 7 rue du Moulin Neuf - 80000 Amiens - France \\ fax : 33322827682 e-mail : name@u-picardie.fr
}

Keywords : tricycle mobile robots, linearization, straight lines, tracking control.

\begin{abstract}
This paper presents a method to track straight lines path with a car-like tricycle vehicle thanks to a straight line tracking controller. The method is validated with several experimental results.
\end{abstract}

\section{Introduction}

For wheeled mobile robots, the easiest path one can plan is composed of several and successive segments of straight lines. Brooks [7] proposes to follow this path by a translation movement on a line until the crossing point with the next segment where it has to stop. Then it turns on itself to obtain the heading orientation of the new straight line segment and moves on it. Path following can then be summarized as several sequences of "Move on a line; Stop; Turn; Move on the next line". So the time to track a path is too long. An approach consisting in joining two crossed segments with arcs of circle was proposed by Nelson and Cox [2]. This method permits to reduce the time needed to track the path in the precedent case. To overcome the discontinuities of path curvature in the above cases, arcs of clothoïds have been used to join two crossed lines by Kanayama and Miyake [8] and by Fleury et al.,[9]. While Nelson [10] solves the problem with polar polynomials, Segovia and Rombaut [11] provide a solution to the continuity problem of curvature between two segments thanks to Bezier's curves. Nevertheless, in what follows the above easiest path will be taken into account.

This paper consists in two parts :

The first part deals with the presentation of a straight line controller designed for an autonomous mobile robot which has three wheels : two free rear wheels with a common axis and one driving and steering front wheel. The driving and steering actuators are respectively a DC series drive and a permanent magnet DC motor. The linearization method proposed in this paper is close to the solution presented by
Sampei et al. [3] for path tracking problems.

The second part is devoted to path tracking with a path composed of several successive or adjacent straight lines with different orientations, the absolute value of the orientation difference between two adjacent lines being less than 90 degrees. A strategy for leaving a line being tracked to track the next one as the robot moves, thanks to the controller above designed is developped. Several experimental results of this control strategy are presented.

\section{Straight line tracking controller}

In this section, a control strategy for straight lines path tracking is presented. The used notations are defined in the following figure :

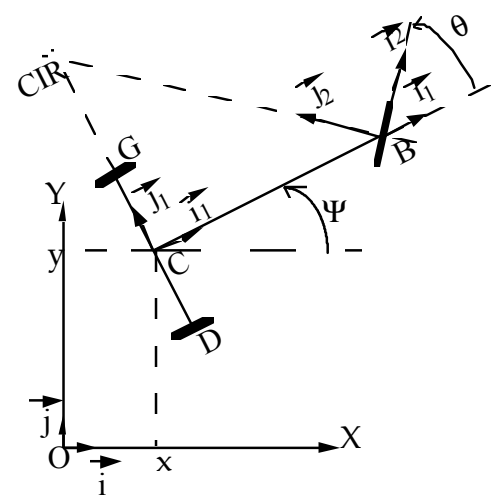

Fig. 1 Robot architecture

$\mathrm{C}_{\mathrm{IR}}$ denotes the intantaneous center of rotation of the vehicle. The following differential equations describe the kinematics of the tricycle robot :

$\left\{\begin{array}{l}\dot{x}=V_{C} \cos \psi \\ \dot{y}=V_{C} \sin \psi \\ \dot{\psi}=\frac{V_{C}}{a} \tan \theta\end{array}\right.$

$\mathrm{V}_{\mathrm{C}}$ is the velocity of the point $\mathrm{C}$, the middle of the rear wheels axis and will be taken as the velocity of the vehicle. 
$\mathrm{a}$ is the distance between the points $\mathrm{C}$ and $\mathrm{B}$. The aim is to design a controller which makes the vehicle follow the $\mathrm{X}$ axis, i.e $\mathrm{y} \rightarrow 0, \psi \rightarrow 0$ and $\theta \rightarrow 0$ when it moves forward. Let a straight line $\mathrm{i}$ in the global and fixed frame $(\mathrm{O}, \mathrm{X}, \mathrm{Y})$ and $\mathrm{O}_{\mathrm{i}}$ its origin whose coordinates in that frame are $\mathrm{X}_{\mathrm{Oi}}$ and $\mathrm{Y}_{\mathrm{Oi}}$ (see figure 2). Let $\Phi_{\mathrm{i}}$ and $\Phi$ denote the orientation angles respectively of the line and of the vehicle with respect to the axis $\mathrm{OX}$ of the frame and $\mathrm{X}$ and $\mathrm{Y}$ the robot's coordinates in the same frame. A direct local frame $\left(\mathrm{O}_{\mathrm{i}}, \mathrm{x}_{\mathrm{i}}, \mathrm{y}_{\mathrm{i}}\right)$ is defined as shown in figure 2 with $\mathrm{i}=1$ for instance.

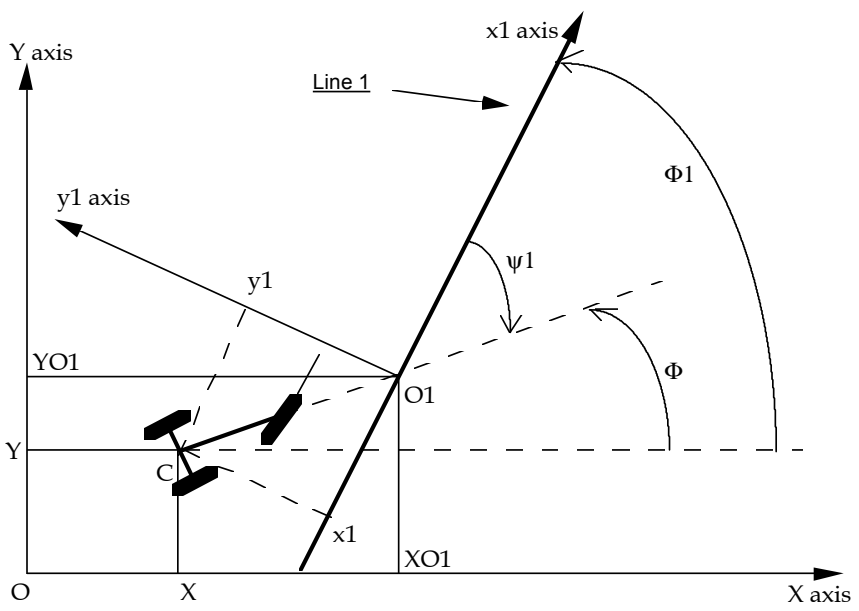

Fig. 2 A straight line in the global frame

The $\mathrm{O}_{\mathrm{i}} \mathrm{x}_{\mathrm{i}}$ axis must be always colinear to the line i. So the vehicle has always to track that axis. Tracking a straight line $i$ will then be equivalent to tracking the corresponding $x_{i}$ axis. Then the coordinates $x_{i}$ and $y_{i}$ of the vehicle, expressed in the local frame, and its orientation $\psi_{i}$ with respect to the $\mathrm{O}_{\mathrm{i}} \mathrm{x}_{\mathrm{i}}$ axis, are obtained as :

$\left(\begin{array}{c}\mathrm{x}_{\mathrm{i}} \\ \mathrm{y}_{\mathrm{i}} \\ \psi_{\mathrm{i}}\end{array}\right)=\left(\begin{array}{ccc}\cos \Phi_{\mathrm{i}} & \sin \Phi_{\mathrm{i}} & 0 \\ -\sin \Phi_{\mathrm{i}} & \cos \Phi_{\mathrm{i}} & 0 \\ 0 & 0 & 1\end{array}\right)\left(\begin{array}{c}\mathrm{X}-\mathrm{X}_{\mathrm{Oi}} \\ \mathrm{Y}-\mathrm{Y}_{\mathrm{Oi}} \\ \Phi-\Phi_{\mathrm{i}}\end{array}\right)$

Considering a new time scale in $\mathrm{x}$, i.e. the distance along the desired path $\left(\mathrm{x}=\mathrm{x}_{\mathrm{i}}\right)$, the equation (1) becomes

$\frac{\mathrm{d}}{\mathrm{dx}}\left(\begin{array}{c}\mathrm{y} \\ \psi\end{array}\right)=\left(\begin{array}{c}\tan \psi \\ 0\end{array}\right)+\left(\begin{array}{c}0 \\ \frac{1}{\mathrm{a} \cos \psi}\end{array}\right) \tan \theta$

while the behaviour of the new time scale $\mathrm{X}$ is given by $\dot{\mathrm{x}}$ in equations (1). The exact linearization of the state equation (3) is obtained by defining the following states variables

$\xi=\left(\begin{array}{l}\xi_{1} \\ \xi_{2}\end{array}\right)=\left(\begin{array}{c}\mathrm{y} \\ \tan \psi\end{array}\right)$ and $\tan \theta=\operatorname{avcos}{ }^{3} \psi$ where $\mathrm{V}$ is the new input variable. In fact the derivative of the new state vector $\xi$ with respect to the new time scale $\mathrm{X}$ gives

$\frac{\mathrm{d}}{\mathrm{dx}}\left(\begin{array}{l}\xi_{1} \\ \xi_{2}\end{array}\right)=\left(\begin{array}{c}\xi_{2} \\ \frac{1}{\mathrm{a} \cos ^{3} \psi} \tan \theta\end{array}\right)$

Writing $\frac{\mathrm{d} \xi_{2}}{\mathrm{dx}}=\mathrm{v}$, we easily obtain

$\tan \theta=\operatorname{av} \cos ^{3} \psi$

Therefore the linearized system is

$\frac{\mathrm{d} \xi}{\mathrm{dx}}=\left(\begin{array}{ll}0 & 1 \\ 0 & 0\end{array}\right) \xi+\left(\begin{array}{l}0 \\ 1\end{array}\right) \mathrm{v}$

for which a state feedback controller

$\mathrm{v}=\left(\begin{array}{ll}\mathrm{f}_{1} & \mathrm{f}_{2}\end{array}\right)\left(\begin{array}{l}\xi_{1} \\ \xi_{2}\end{array}\right)$

can be designed, using pole placement, for instance, to ensure the stability of the system. $f_{1}$ and $f_{2}$ are constants to be defined. If the poles $p_{1}$ and $p_{2}$ are chosen then $f_{1}=-p_{1} p_{2}$ and $f_{2}=p_{1}+p_{2}$. In fact the closed loop linearized system is equivalent to the second order differential equation (9) in $y\left(y=y_{i}\right)$

$y^{\prime \prime}-f_{2} y^{\prime}-f_{1} y=0$

$y^{\prime}$ and $y^{\prime \prime}$ denoting the first and the second derivatives of $y$ with respect to the new time scale $X$. Its cross over pulsation $\omega_{\mathrm{n}}$ and its decay coefficient $\mathrm{Z}$ are given in the relation (10).

$\left\{\begin{array}{l}\omega_{\mathrm{n}}=\sqrt{-\mathrm{f}_{1}} \\ \mathrm{z}=\frac{1}{2} \frac{\mathrm{f}_{2} \sqrt{-\mathrm{f}_{1}}}{\mathrm{f}_{1}}\end{array}\right.$

The choice of the stable poles for the system can be done by giving a value to $\mathrm{Z}$. This implies

$\left\{\begin{array}{l}\mathrm{f}_{1}<0 \\ \mathrm{f}_{2}=-\mathrm{z} \sqrt{-4 \mathrm{f}_{1}}\end{array}\right.$

In critical case (i.e. $z=1$ ), giving for instance, a value to $f_{1}$ is a manner to determine a real double poles $\left(\mathrm{p}_{1}=\mathrm{p}_{2}\right)$ and the cross over pulsation of the system. The steering speed is obtained thanks to the equation (12): 
$\dot{\theta}=\mathrm{aV}_{\mathrm{C}}(\mathrm{F}+\mathrm{G}) \cos ^{2} \theta \cos ^{2} \psi$

with $\left\{\begin{array}{l}F=f_{1} \sin \psi \cos \psi+\frac{f_{2}}{a} \frac{\tan \theta}{\cos \psi} \\ G=-3 \frac{\tan \theta}{a}\left(f_{1} y+f_{2} \tan \psi\right) \sin \psi\end{array}\right.$

Because of the definitions of the coordinates transformation (4) and the feedback (6), this control is defined only if $\psi, \theta$ and $y$ belong respectively to $] \pi / 2, \pi / 2[$, $] \pi / 2, \pi / 2[$ and ]$-\infty, \infty[$.

The simulations results of this controller for $\mathrm{z}=1$, $\mathrm{f}_{1}=-4 \mathrm{~m}^{-2}$ (i.e. $\mathrm{f}_{2}=-4 \mathrm{~m}^{-1}$ ) and a constant speed $\mathrm{V}_{\mathrm{C}}=0.15 \mathrm{~m} / \mathrm{s}$ are shown in the figure 3 . The path to follow in the global frame is expressed as $\mathrm{Y}=-\sqrt{3} \mathrm{X}+4$. Initial conditions are $\xi_{1}=-10 \mathrm{~m}, \xi_{2}=\tan \frac{\pi}{3}$ and $\mathrm{x}=-10 \mathrm{~m}$. The robot follows the desired straight line.

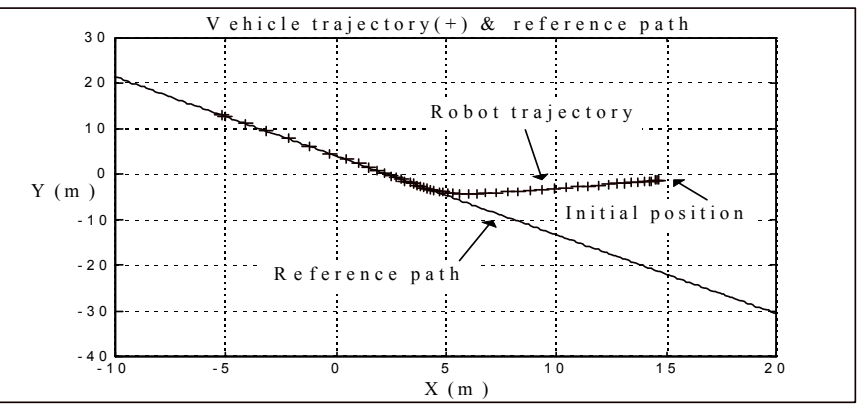

Fig. 3.a : A straight line tracking - simulation result

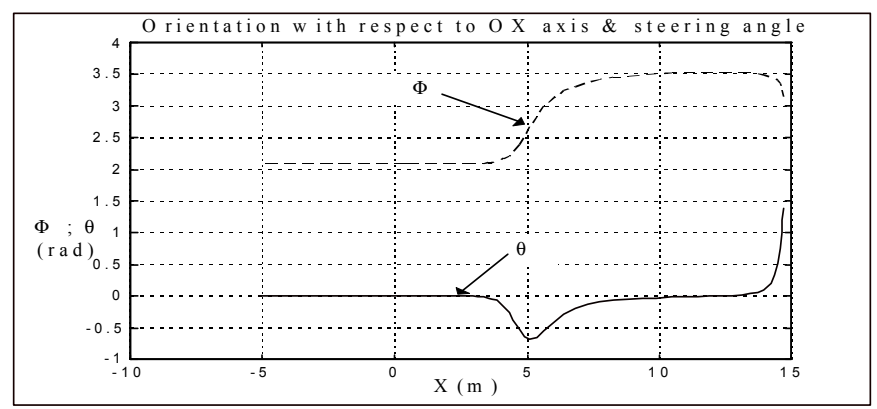

Fig. 3.b : Orientation $(\Phi)$ and steering $(\theta)$ angles

\section{Path tracking strategy}

\section{3-1 Problem statement}

The absolute value of the orientation's angle difference $\Delta \Phi=\Phi_{i+1}-\Phi_{i}$ between the lines $\mathrm{i}+1$ and $\mathrm{i}$ is assumed less than $\pi / 2$ radians. The origin of the first line is chosen everywhere and the origin of the line $\mathrm{i}(\mathrm{i}>1)$ is the crossing point between lines $\mathrm{i}$ and $\mathrm{i}-1$.
Let the path in figure 4 be the reference one and $\theta_{i}$ the needed steering angle to track the straight line $\mathrm{i}$ while the robot is at any position in the global frame respecting the validity domain defined before for the controller.

With the system (2) the robot's position in the local frame of any line $\mathrm{i}$ is easily obtained. For instance, on the figure $\theta_{1}=0, \quad \psi_{1}=0, \quad \mathrm{x}_{1}=\mathrm{O}_{1} \mathrm{C}, \quad \mathrm{y}_{1}=0 \quad$ and $\quad \mathrm{y}_{2}=\mathrm{O}_{2} \mathrm{D}$. Considering that the vehicle is tracking the line 1 and has the position shown in the figure 4 , at what position $\mathrm{x}_{1}$ it will stop tracking this line and begin to join the next one (line 2)? Sampei et al [3] sayed that at least, the steering angle must be continuous i.e. $\theta_{2}=\theta_{1}$. But this continuity condition can be obtained in several positions $x_{1}$ for several values of $f_{1}$ when the decay coefficient is fixed. As seen before, to give a value to $f_{1}$ is equivalent to choose the dynamics of the robot.

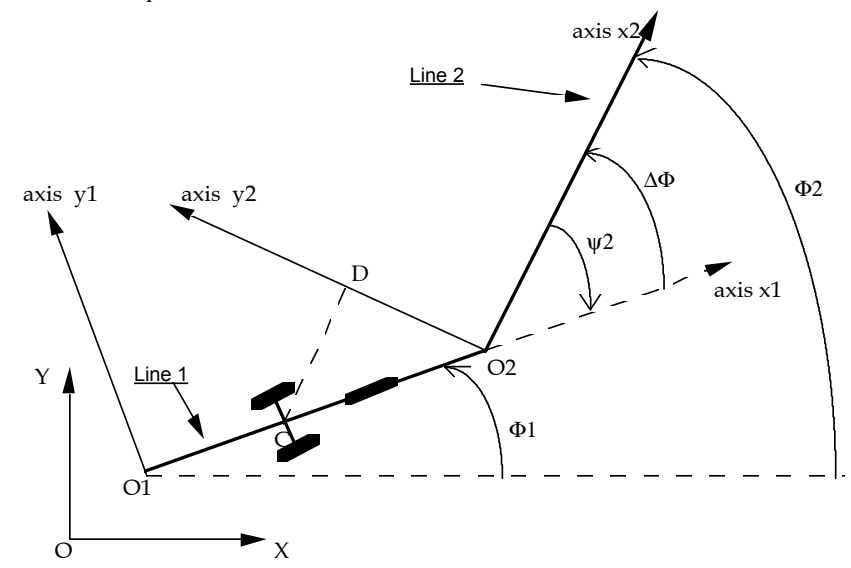

Fig. 4 : A path composed with two straight lines

One can then work in this sense considering the continuity of the steering angle. Here the strategy which permits to track a line as long as possible and indicates exactly the position from which the robot will stop tracking a current line and begin to join the next one is presented.

\subsection{Tracking strategy}

At the position where the line changes, the relations (6) and (8) and the continuity of the steering angle give

$$
\left\{\begin{array}{l}
\theta_{1}=\tan ^{-1}\left[\mathrm{a}\left(\mathrm{f}_{1} \mathrm{y}_{1}+\mathrm{f}_{2} \tan \psi_{1}\right) \cos ^{3} \psi_{1}\right] \\
\theta_{2}=\tan ^{-1}\left[\mathrm{a}\left(\mathrm{f}_{1} \mathrm{y}_{2}+\mathrm{f}_{2} \tan \psi_{2}\right) \cos ^{3} \psi_{2}\right] \\
\theta_{2}=\theta_{1}
\end{array}\right.
$$

Then, while tracking the first straight line (i.e. $\theta_{1}$ is calculated), the steering angle $\theta_{2}$ needed to follow the next one, the line 2, must also be computed. At the configuration where $\theta_{2}=\theta_{1}$, the robot will stop tracking the first line and start to follow the second and next straight line. To simplify this idea, we only consider the path whose first straight line 
is colinear to the longitudinal axis of the vehicle i.e. the first line has the initial orientation of the robot in the global frame with the robot exactly on the line as shown in figure 4 . With this in mind, in figure $4, \psi_{2}=\Phi_{1}-\Phi_{2}=-\Delta \Phi$. As $\psi_{1}=0$ and $\mathrm{y}_{1}=0$ then $\theta_{1}=0$ and $\mathrm{y}_{2}$ can be expressed as

$y_{2}=-\frac{f_{2}}{f_{1}} \tan \psi_{2}=-z \frac{\sqrt{-4 f_{1}}}{f_{1}} \tan \Delta \Phi$

The exact position from which the robot will stop tracking a current line (line 1) and begin to join the next (line 2) can now be indicated. This will be done without need to compute the steering angles.

Except the last line of the path, we impose a security distance $d_{i}$ (see figure 5) from the end of each line $i$, with in mind that the end of the line $i$ is the origin of the line $i+1$. Then the needed change on the straight line $i$ will occur when $\mathrm{x}_{\mathrm{i}}=\mathrm{O}_{\mathrm{i}} \mathrm{O}_{\mathrm{i}+1}-\mathrm{d}_{\mathrm{i}}$ i.e. $\mathrm{d}_{\mathrm{i}}=\mathrm{CO}_{\mathrm{i}+1}$. With $\Delta \Phi_{\mathrm{i}}=\Phi_{\mathrm{i}+1}-\Phi_{\mathrm{i}}$ one has

$\mathrm{y}_{\mathrm{i}+1}=\mathrm{d}_{\mathrm{i}} \sin \Delta \Phi_{\mathrm{i}}=-\mathrm{d}_{\mathrm{i}} \sin \psi_{\mathrm{i}+1}$

Then, on figure 5 this relation (16) becomes

$\mathrm{y}_{2}=\mathrm{d}_{1} \sin \Delta \Phi_{1}=-\mathrm{d}_{1} \sin \psi_{2}$

The relations (14) and (17) imply

$$
d_{1}=-z \frac{\sqrt{-4 f_{1}}}{f_{1} \cos \psi_{2}}
$$

With the robot on the line $\mathrm{i}$, the general expression of the security distance is given by the relation (19)

$$
d_{i}=-z \frac{\sqrt{-4 f_{1}}}{f_{1} \cos \psi_{i+1}}=-z \frac{\sqrt{-4 f_{1}}}{f_{1} \cos \Delta \Phi_{i}}
$$

The above variables are shown in the figure 5 .

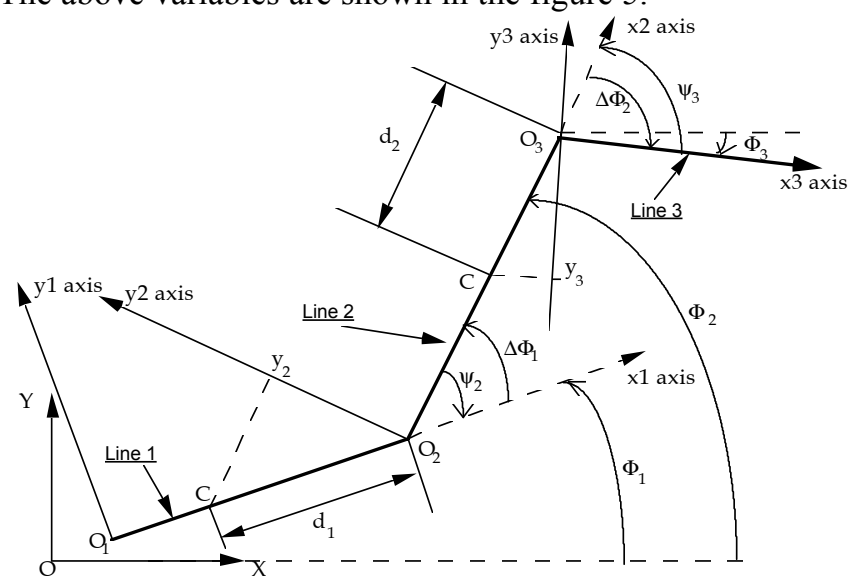

Fig. 5 : Path with the security distances $d_{1}$ and $d_{2}$
The relation (19) shows that for fixed values of $\mathrm{Z}$ and $\mathrm{f}_{1}$, the security distance $d_{i}$ is a function of $\Delta \Phi_{i}$ the orientation difference between the next line to join and the line actually tracked.

Two cases will be presented :

Case 1: The lines are long enough. Here, all straight lines of the reference path are sufficiently long to permit the robot which is moving on a line $\mathrm{i}$ to reach the security distance $d_{i}$, then, join the line $i+1$, moves on it before reaching the corresponding security zone $d_{i+1}$ from which it starts to join the next straight line and so on.

Case 2 : Some lines of the path are not long enough. A straight line can be shorter than the corresponding security distance (computed from equation 19) needed to perform the continuity of the steering angle i.e. the vehicle is already in the security zone of the straight line it has to track. In this case, the robot has to directly follow the next line.

\subsection{The tracking strategy algorithm}

As written before, at initial time, the robot will be on the first line with $\Phi=\Phi_{1}$. The algorithm below takes into account the above presented cases to obtain the correct continuity of the steering angles while tracking a desired path. For given values of $\mathrm{Z}$ and $\mathrm{f}_{1}$, the algorithm to track a path of $\mathrm{N}$ straight lines $(\mathrm{N}>1)$ is as follows :

1-) Give a low value of the moving speed $V_{C}$

2-) $\mathrm{i}=1$ ( $\mathrm{i}$ is the number of the line).

3-) Compute $\Delta \Phi_{\mathrm{i}}=\Phi_{\mathrm{i}+1}-\Phi_{\mathrm{i}}$

4-) Compute the security distance $d_{i}$ thanks to (19).

5-) Compute $x_{i}, y_{i}$ and $\psi_{i}$ thanks to the relation (2)

5-1) If $\mathrm{x}_{\mathrm{i}}<\mathrm{O}_{\mathrm{i}} \mathrm{O}_{\mathrm{i}+1}-\mathrm{d}_{\mathrm{i}}$ then

* Track the line $i$ (i.e.- Compute $\theta_{i}$ and $\dot{\theta}_{i}$ - Reach the angle $\theta_{i}$ with the steering speed $\dot{\theta}_{i}$ - Move the robot with the constant speed $\mathrm{V}_{\mathrm{C}}$ ) and go to 5

5-2) Else

* Increment $\mathrm{i}$

* If $\mathrm{i}=\mathrm{N}$ then track the line $\mathrm{i}$ until its end point and stop.

Else go to 3.

\section{Path tracking experimental results}

The above strategy has been implemented on an industrial tricycle [6] with low values of the moving speed $\left(\mathrm{V}_{\mathrm{C}}<0.3 \mathrm{~m} / \mathrm{s}\right)$ and for a decay coefficient $\mathrm{z}=1$. The obtained experimental results are good as shown next, in the figures 7 , 8 and 9 , for $\mathrm{f}_{1}=-4 \mathrm{~m}^{-2}, \mathrm{~V}_{\mathrm{C}}=0.15 \mathrm{~m} / \mathrm{s}$ and for three cases. These results use only basic sensors : an incremental encoder for the displacement, a tachogenerator for the moving speed, a tachogenerator for the steering speed and a potentiometer 
for the steering angle. Their implantation on the robot is summarized in figure 6 .
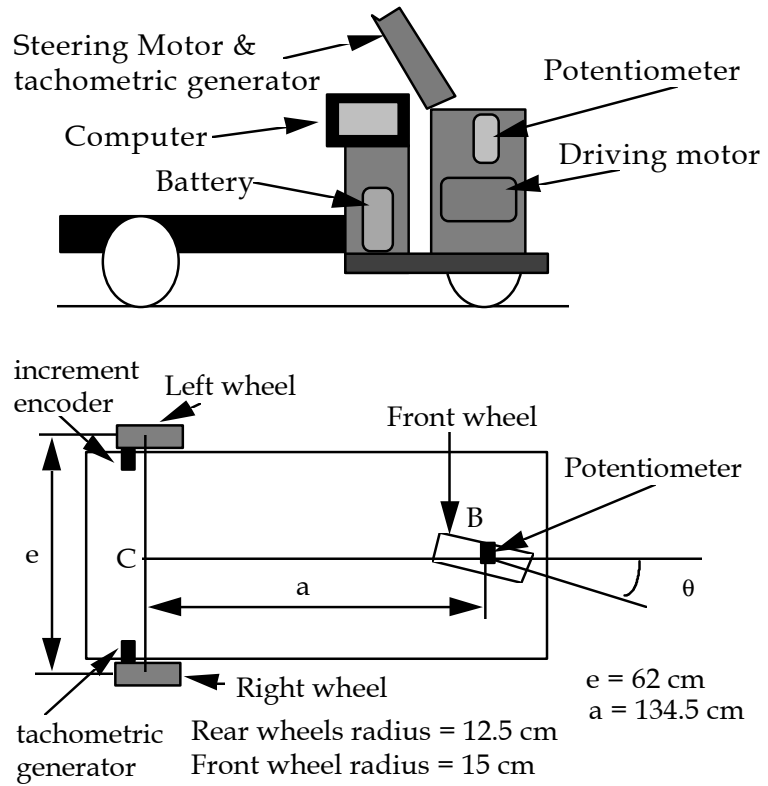

Fig. 6 : Vehicle sensors

case 1) The desired path is composed with three lines whose equations in the global frame $(\mathrm{O}, \mathrm{X}, \mathrm{Y})$ are in order $\mathrm{Y}_{1}=0, \mathrm{Y}_{2}=\sqrt{3}(\mathrm{X}-4)$ and $\mathrm{Y}_{3}=4$. Calling $(\mathrm{X}, \mathrm{Y}, \Phi)$ the position of the robot in that frame, the initial and desired final positions of the vehicle are respectively $(0,0,0)$ and $(10.3 \mathrm{~m}, 4 \mathrm{~m}, 0)$. The experimental results are in the figure 7 .

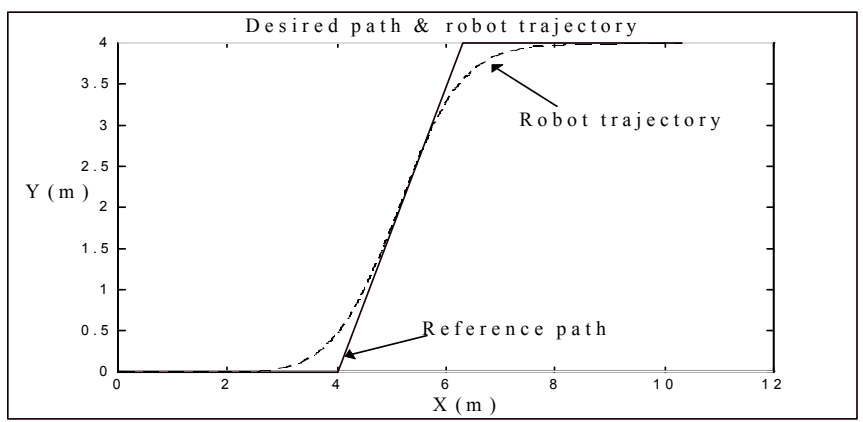

Fig. 7.a : Reference path and robot trajectory

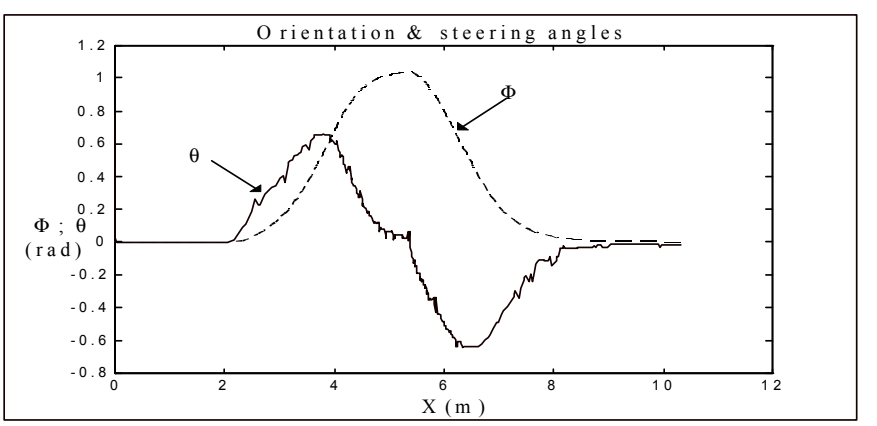

Fig. 7.b : Orientation and steering angles
Case 2) The reference path is composed with four lines which are in order $\mathrm{Y}_{1}=0, \quad \mathrm{Y}_{2}=\sqrt{3}(\mathrm{X}-7)$, $\mathrm{Y}_{3}=-\sqrt{3}(\mathrm{X}-7)+4$ and $\mathrm{Y}_{4}=4$. Initial and desired final positions of the vehicle are respectively $(0,0,0)$ and $(3 \mathrm{~m}, 3 \mathrm{~m}, \pi \mathrm{rad})$. The results are in the figure 8 .

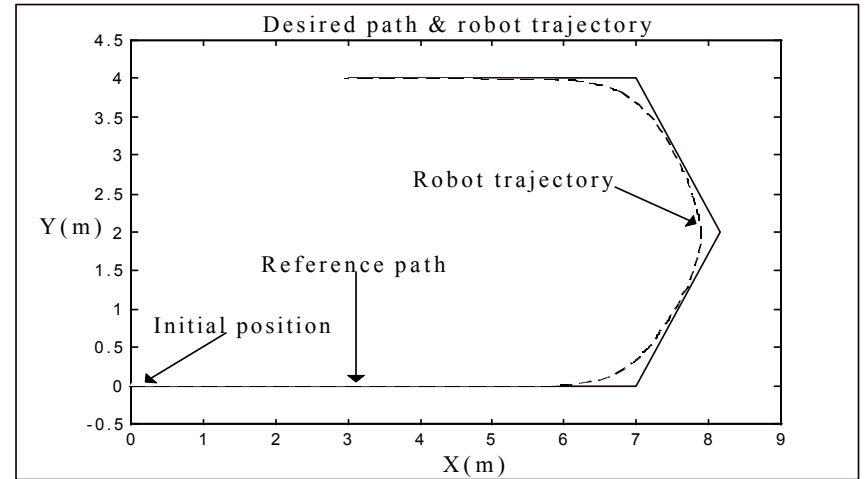

Fig. 8.a : Desired path and robot trajectory

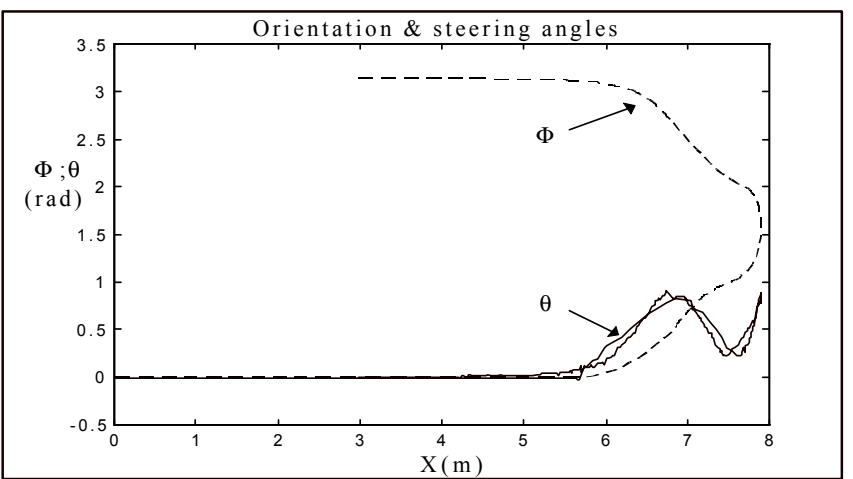

Fig. 8.b : Orientation and steering angles

Case 3) Case of a short first straight line. The path is composed with three lines $Y_{1}=0, Y_{2}=\sqrt{3}(X-0.2)$ and $\mathrm{Y}_{3}=4$. The initial and desired final positions of the robot are respectively $(0,0,0)$ and $(6.5 \mathrm{~m}, 4 \mathrm{~m}, 0)$. The obtained results are shown in the figure 9.

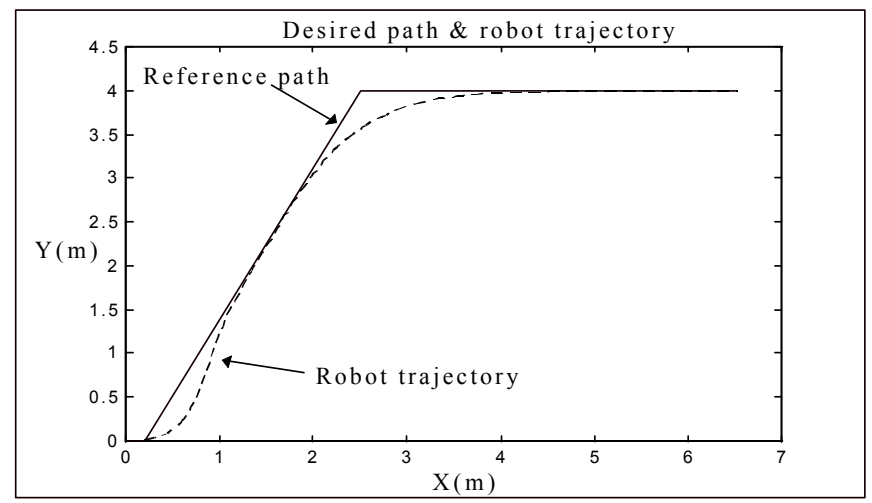

Fig. 9.a : Reference path and robot trajectory 


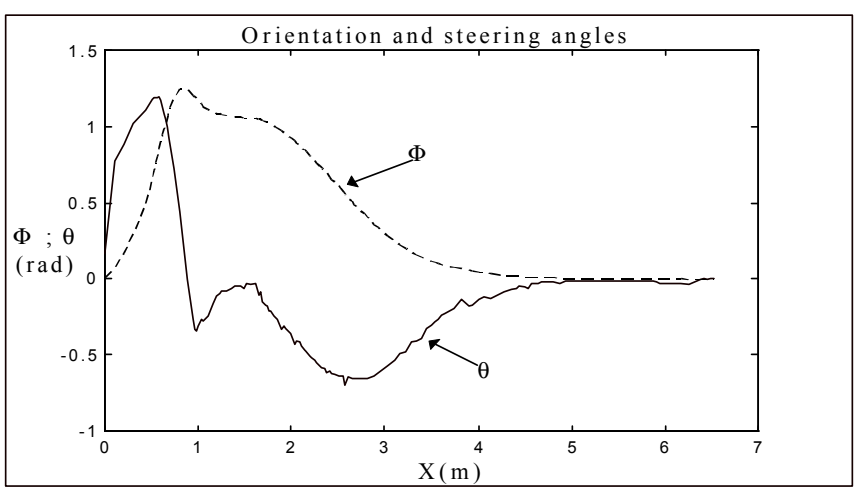

Fig. 9.b : Orientation and steering angles

The proposed tracking strategy developped before and using the straight line controller is very satisfactory.

At this stage it is important to note the sensitivity of the the dynamic response to the choice of $f_{1}$. In fact for the critical case $(z=1)$, as the absolute value of $f_{1}$ increases, the vehicle response becomes faster to reach the reference straight line. This observation can be seen in figure 10 .
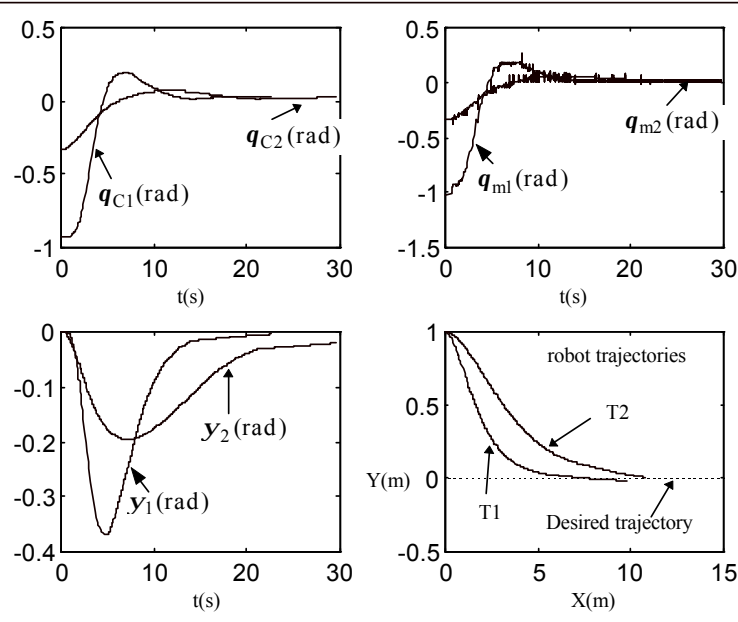

Fig. 10 : Experimental results for different values of $f_{1}(z=1)$

Two experimental results are shown for the same moving speed $\mathrm{V}_{\mathrm{C}}$ and initial position $(0,1 \mathrm{~m}, 0)$ of the robot in the global frame. In both cases the desired path is the $\mathrm{X}$-axis. The first case is for $f_{1}=-1 \mathrm{~m}^{-2}$ and the results are indexed with 1 i.e. $\left(\theta_{\mathrm{C} 1}, \theta_{\mathrm{ml}}, \psi_{1}, \mathrm{~T}_{1}\right)$ in the figure. $\theta_{\mathrm{C} 1}$ and $\theta_{\mathrm{ml}}$ are respectively the computed (or desired) and the measured (or effective) steering angles. $T_{1}$ is the trajectory of the robot. The other case is for $\mathrm{f}_{1}=-0.25 \mathrm{~m}^{-2}$ and the results are indexed with 2 .

\section{Conclusion}

Experimental results have been presented for the robust control of an industrial nonlinear tricycle robot. Dynamics effects, as the vehicle inertia, the tyres analysis for instance, have been neglected by choosing low moving reference speed $\mathrm{V}_{\mathrm{C}}$. The obtained performances are very satisfactory and have been performed using the straight line controller.

\section{References}

[1] A. Hemami, M. G. Mehrabi, and R.M.H. Cheng, "A New Control Strategy for Tracking in Mobile Robots and AGVs," IEEE pp. 1122-1127, (1990).

[2] Nelson W.L., I.J. Cox, " Local Path Control for an Autonomous Vehicle" IEEE Int. Conf. on Robotics and Automation, Vol 3, pp 1504-1510. (1988).

[3] Sampei M. et al " Arbitrary Path Tracking Control of articulated Vehicles Using Nonlinear Control Theory ". IEEE Transactions. on Control Systems Technology. Vol 3, pp 125-131, (1995).

[4] C. Samson, " Control of Chained Systems, Application to Path Following and Time-Varying PointStabilization of Mobile Robots, " IEEE Transactions on Automatic Control, Vol. 40, N 1, pp 64-77, (1995).

[5] André KAMGA, "Instrumentation et Commande d'un Robot Mobile Industriel" $\mathrm{PhD}$ Thesis, Université de Picardie Jules Verne, Amiens, France, October (1996).

[6] Charlatte's society, " Documentation technique sur le Tricycle multiservice TMS T10,"(1990).

[7] Brooks R., " Solving the Find-Path Problem by Good Representation of Free Space, " IEEE Transactions on Systems, Man and Cybernetics, Vol SMC-13, $\mathrm{N}^{\circ}$ 3, (1983).

[8] Kanayama Y. and Miyake N., " Trajectory generation for Mobile Robots, " In : G. Giralt O. Faugeras, editor, Robotics Research 3, MIT Press, (1986).

[9] Fleury S., Soueres P., Laumond J.P., Chatila R,. "Primitives for Smoothing Mobile Robot Trajectories". Proc. IEEE Int. Conf. on Robotics and Automation. Atlanta Georgia, (1993).

[10] Nelson W., "Continuous-Curvature Paths for Autonomous Vehicles". Proc. IEEE Int. Conf. on Robotics and Automation, Vol 3, (1989).

[11] Segovia A., Rombaut M. Continuous curvature path finding for a mobile robot in a closed environment. 1st IFAC Int. Workshop on intelligent Autonomous Vehicles, Southampton, (1993).

[12] B. Steer, "Trajectory Planning for a Mobile Robot, " The international Journal of Robotics Research, Vol 8, $\mathrm{N}^{\circ}$ 5, pp 3-14, (1989). 